\title{
VP Bandwidth Management with Dynamic Connection Admission Control in ATM Networks
}

\author{
Yoshinori Kawamura Iliroshi Saito \\ NTT Telecommunication Networks Laboratories \\ 3-9-11, Midori-cho, Musashino-shi, Tokyo 180, Japan \\ Fax: +\$1 422593290 F-mail: kawamura Ghashi.ntt.jp
}

\begin{abstract}
This paper discusses a VP bandwidth management with connection admission control (CAC:) based on traffic measurements, i.e., Dynamic C:AC, in ATM networks. Dynamic CAC initially assigns a bandwidth equivalent to its peak cell rate for a new connection. and then corrects it after traffic measurement. So Dynamic CAC has the advantage of statistical multiplexing gain, even when only the peak cell rate is notified to the network. To use Dynamic CAC effectively. however, it is important to appropriately set a parameter that specifies the weight of traffic characteristics in deciding ad mission. This parameter is called a smoothing parameter. This paper develops a method of designing the smoothing parameter.
\end{abstract}

\section{Introduction}

In Asynchronous Transfer Mode (ATM) networks, a fixed size block called a cell is used to transport varieties of information and there are no complicated controls such as retransmission. Therefore, ATM networks can provide high-speed broadband communication. In addition. they can assign network resources flexibly to each connection because the necessary number of cells are generated according to the amount of information to be conveyed. However. due to these characteristics of ATM networks, new traffic control techniques are required in ATM networks. The two fundamental traffic controls in ATM networks are Connection Admission Control (CAC) and Usage Parameter C'ontrol (UPC). In CAC (Figure 1), a user notifies the characteristics of a Virtual Channel (VC) at the connection setup, called traffic descriptor, and the network judges whether or not there are enough resources for the VC, if there are, it accepts the VC. In UPC, the network monitors how the notified traffic characteristics conform to the actual ones of the $\mathrm{VC}$ and imposes a penalty such as discarding or tagging cells if they do not conform.

One of the main problems with resource management based on CAC: and UPC: is the predictability of traffic characteristics. The ITU-T recommendation[1] states that one of the traffic parameters describing traffic descriptor must be the peak cell rate, and that other parameters, such as sustainable cell rate and burst tolerance, will be standardized. However, many services such as high-speed data communication cannot specify traffic 
characteristics other than the peak cell rate at the time of connection setup. Thus, economical communication cannot be provided for such services when enough bandwidth to accommodate the peak cell rate is assigned for the whole duration of the connection. One technique for overcoming this situation is Dynamic CAC. This CAC: measures the number of cells arriving at each $V P$ and judges the admission of a new $V C$ ? based on that measurement and on the peak cell rate of the VC (Fig.2). Since Dynamic C.AC uses the traffic measurement, $V C$ 's can be statistically multiplexed even when the only peak cell rate is notified to the network. To use dynamic CAC effectively, it is important to appropriately set a parameter that specifies the weight of traffic measurement in deciding admission. This parameter is called a smoothing parameter. The case for a homogeneous traffic conditions is discussed in Ref.[2]. The present papaer extends the method of designing the smoothing parameter to heterogeneous traffic conditions.

\section{Dynamic CAC}

In this section, we describe Dynamic CAC: and its smoothing parameter. Although Dynamic CAC: is applicable even when the traffic descriptor includes parameters other than the peak cell rate. we consider the case where traffic descriptors have only the peak cell rate according to the 1992 ITU-T recommendation in this paper. In addition, we assume that UPC: works properly and that the arrival peak cell rates from users are always kept. below the notified ones.

\subsection{Algorithm of Dynamic CAC}

Throughout this paper, we denote the VP bandwidth by $C$, the output buffer size by $K$ and the length of the cell by $l$. The traffic measurement scheme for Dynamic CAC: is as follows. We count the cells arriving at the output buffer during a fixed-length period $w$ which is definded by $w=r \cdot l / C$. We call this period a measurement period. and furthermore we call the period consisting of $N$ measurement periods the update period. We denote the measured frequency distribution in these $N$ periods at the th update period by $q(k . t)(k=0.1, \cdots)$. Set $\mathbf{q}(t)={ }^{T}(q(0, t), q(1, t), \cdots)$, where ${ }^{T} A$ for a matrix $A$ means its transposed matrix. The following procedure estimates the distribution of the number of arriving cells. We write this estimated distribution as $\hat{\mathbf{p}}(t)$.

(1) If the number of existing VCs does not change during an update period,

$$
\hat{\mathbf{p}}(t+1)=\alpha \mathbf{q}(t)+(1-\alpha) \hat{\mathbf{p}}(t) . \quad(0 \leq \alpha \leq 1)
$$

This parameter $\alpha$ is said a smoothing parameter.

(2) When a new class- $j \mathrm{VC}$ request is connected in the $t$ th update interval such that the maximum number of cells arriving (MNA) during the fixed-length interval $u$ from the new connection is $R_{j}$, the renewing procedure is

$$
\hat{\mathbf{p}}(t+1)=\Phi_{j}[\hat{\mathbf{p}}(t)] .
$$


Here, for $\hat{\mathbf{p}}(t)={ }^{T}(\hat{p}(0, t), \hat{p}(1, t), \cdots)$, we define $\Phi_{j}[\hat{\mathbf{p}}(t)]$ by

$$
\Phi_{j}[\hat{\mathbf{p}}(t)]=\left(\begin{array}{c|l}
0 \\
\vdots \\
0 & \vdots \\
\hat{p}(0, t) & R_{j} \\
\hat{p}(1, t) & R_{j}+1 \\
\vdots & R_{j}+2 \\
\hat{p}\left(R_{T}-R_{j}, t\right) & R_{T}+1 \\
0 & R_{T}+2 \\
\vdots & \vdots
\end{array}\right.
$$

where $R_{T}$ is the sum of all MNAs in all the connected VC:s (including the new one). This equation means that the number of cells arriving from the new $V C$ is a priori assumed to be $R_{j}$. The value $R_{j}$ is given by the peak cell rate described by a user.

(3) When a VC: connection with MNA $=R_{j}$ completes its service and is released during the th update period, we use the following procedure:

$$
\hat{\mathbf{p}}(t+1)=\Omega_{j}[\hat{\mathbf{p}}(t)] .
$$

Here, for $\hat{\mathbf{p}}(t)={ }^{T}(\hat{p}(0, t), \hat{p}(1, t), \cdots)$, we define $\Omega_{j}[\hat{\mathbf{p}}(t)]$ by

$$
\Omega_{j}[\hat{\mathbf{p}}(t)]=\left(\begin{array}{c|l}
\hat{p}(0, t) \\
\hat{p}(1, t) \\
\vdots \\
\hat{p}\left(R_{T}-R_{j}-1, t\right) \\
\sum_{\hat{k}=R_{T}-R_{j}}^{\infty}(k, t) \\
0 \\
\vdots
\end{array}\right) \begin{aligned}
& 1 \\
& R_{T}-R_{j} \\
& R_{T}-R_{j}+1 \\
& R_{T}-R_{j}+2 \\
& \vdots
\end{aligned}
$$

where $R_{T}$ is the sum of all MNAs in all the connected VCs (including the released one).

When there is no estimation error, the estimate of the upper bound of the cell loss ratio can be obtained from this distribution $\hat{\mathbf{p}}(t)$ by applying Ref.[1],[3],[4]. Dynamic C.AC decides whether a new connection request during the $(t+1)$ th update period may be accepted or not using the estimate $\hat{B}^{(j)}(t+1)$, which is the estimate of the upperbound of the cell-loss-ratio assuming it is accepted. It is defined by

$$
\hat{B}^{(j)}(t+1)=\frac{\sum_{k=0}^{\infty} \max \{0, k-r\} \hat{p}_{+}^{(j)}(k, t)}{\sum_{k=0}^{\infty} k \hat{p}_{+}^{(j)}(k, t)}
$$

where $\hat{p}_{+}^{(i)}(k, t)$ is the $(k+1)$ th compornent of the vector $\Phi_{j}[\hat{\mathbf{p}}(t)], r \leq(K+1) / 2$.

\subsection{Smoothing parameter}

Dynamic C:AC: corrects the bandwidth assigned according to a source traffic descriptor by using the measured number of arriving cells and the smoothing parameter $\alpha$. When $\alpha$ is 0 , the $\mathrm{CAC}$ assigns the peak-cell-rate bandwidth to each $\mathrm{VC}$. This results in low utilization, 
but it can attain high quality. When $\alpha$ is nearly 1 , Dynamic C.AC may misjudge whether a new $V C$ request is acceptable or not. because the distribution $\hat{\mathbf{p}}(t)$ is greatly influenced by the last measurement.

However, in general, it is expected that Dynamic CAC supports QOS requirements sufficiently for the following reason. First, VP bandwidth is much larger than VC: bandwidth, so rapid changes in a few users' traffic characteristics do not make the QOS worse. Second, the estimate $\hat{B}^{(i)}(t+1)$ in Eq.(1) is upper-bounded. And even if cell loss has occurred frequently, the congestion control in Ref.[5] works effectively, so network performance is not so bad. In order to gain the advantages of statistical multiplexing, it is important to consider how to design the smoothing parameter. In this paper, we describe a method for designing the smoothing parameter for heterogeneous traffic conditions. We consider the error probability that a new VC. request is accepted when the VP is saturated with VCs. This design method improves statistical multiplexing limiting the error probability to below a certain fixed level.

\subsection{Smoothing parameter design method}

One of the useful characteristics of Dynamic C:AC! is that it operates well without assuming any particular cell arrival process model. However, in general, the most suitable smoothing parameter for improving statistical multiplexing depends on the traffic characteristics of $V C$ 's in the VP; for example, the arrival rate, the holding time, the peak cell rate, and the average cell rate. It is not realistic to modify the smoothing parameter in real time as these these traffic characteristics change. In this paper, we consider a method of designing the smoothing parameter for Dynamic C.AC on a long time scale, such as a day, week or month. Therefore, in order to design the smoothing parameter, we consider the cell arrival process model from the design viewpoint. Note that $\hat{\mathbf{p}}(t)$ and the estimate $\hat{B}^{(j)}(t+1)$ have a certain values when Dynamic CAC: has done, so they are not ramdom variables. However, from a design viewpoint, they are random variables depending on the traffic characteristics and the parameters of Dynamic CAC, because the number of arriving cells, the setup times, and the released time of VCs change statistically.

We denote the cell loss rate objective by $B_{0}$, and assume that $m$ class VC:s accumulate in the VP.

\subsection{Traffic Model}

Dynamic CAC: decides whether to accept or reject a new VC: request by considering its anticipated traffic characteristics, the $\mathrm{QOS}$ requirements of $\mathrm{VC} \cdot \mathrm{s}$, and the evaluated cellloss-ratio at the output buffers of the switch. If we regard the VP as a server, a cell transfer as a service, and a cell as a customer, we can consider the output part of the switch to be a single server queue system with finite buffers.

\subsection{Formulation}

First, let us formulate the conditions under which we will design an appropriate smoothing parameter. 


\subsubsection{Saturation of VP}

Let $\mathbf{n}=\left(n_{1}, n_{2}, \cdots, n_{m}\right)$ be a vector such that its $j$ th component $n_{j}$ means the number of class- $j$ VC:s connected on the VP simultaneously, and let $q(k: \mathbf{n})$ be the probability that the measured number of cells arriving during the fixed-length interval $w$ is $k$. Ref.[4] shows that if $\mathbf{n}$ satisfies

$$
\frac{\sum_{k=0}^{\infty} \max \{0, k-r\} q(k: \mathbf{n})}{\sum_{k=0}^{\infty} k q(k: \mathbf{n})} \leq B_{0},
$$

the cell-loss-ratio objective $B_{0}$ is guaranteed. Let the set consisting of $\mathbf{n}$ satisfying this equation be $V$, and a subset $V_{j}$ of $V$ be defined by

$$
V_{j}=\left\{\mathbf{n} \in V:\left(n_{1}, \cdots, n_{j}+1, \cdots, n_{m}\right) \notin V\right\} .
$$

We say that the VP is saturated for class- $j$ VCs when $\mathbf{n}$ belongs to $V_{j}(j=1, \cdots, m)$.

\subsubsection{Estimate of the Cell Loss Ratio}

To clarify the dependence of $\mathbf{n}$ upon the estimate $\hat{B}^{(j)}(t+1), \hat{\mathbf{p}}(t)$, we rewrite $\hat{B}^{(j)}(t+1)$ and $\hat{\mathbf{p}}(t)$ as $\hat{B}^{(j)}(t+1: \mathbf{n})$ and $\hat{\mathbf{p}}(t: \mathbf{n})$ respectively. Note that

$$
\hat{B}^{(j)}(t+1: \mathbf{n})=\frac{\sum_{k=0}^{\infty} \max \{0, k-r\} \hat{p}_{+}^{(j)}(k, t: \mathbf{n})}{\sum_{k=0}^{\infty} k: \hat{p}_{+}^{(j)}(k, t: \mathbf{n})} .
$$

In general the limits $\lim _{t \rightarrow \infty} \hat{B}^{(j)}(t+1: \mathbf{n})$ and $\lim _{t \rightarrow \infty} \hat{\mathbf{p}}(t)$ do not exist at all times.

However, it is natural to assume that the process $\{\hat{\mathbf{p}}(t)\}$ becomes embedded Markovian process, considering the process shortly after a VC. setup request arrives. Namely, if $t_{n}$ is the update period during which the $n$th class- $j$ VC setup request arrives, then the process $\left\{\hat{\mathbf{p}}\left(t_{n}\right)\right\}_{n=1}^{\infty}$ is Markovian. Therefore we assume the $\operatorname{limits}_{\lim _{t_{n} \rightarrow \infty}} \hat{\mathbf{p}}\left(t_{n}: \mathbf{n}\right)$ and $\lim _{t_{n} \rightarrow \infty} \hat{B}^{(j)}\left(t_{n}+1: \mathbf{n}\right)$ do exist. Next, consider the estimate $\hat{B}^{(j)}(\mathbf{n})$, which is given by

$$
\hat{B}^{(j)}(\mathbf{n}) \sim_{d} \lim _{t_{n} \rightarrow \infty} \hat{B}^{(j)}\left(t_{n}+1: \mathbf{n}\right)
$$

where $A \sim{ }_{d} B$ means that two random variables $A$ and $B$ have the same probability distribution. To evaluate the error probability which is the probability that the request of a VC of new class- $j$ is accepted even when the VP is saturated for class- $j$ VCs (Fig.4), we analyze the relationship between the smoothing parameter and a percentile point of the distribution of $\hat{B}^{(j)}(\mathbf{n})$ in the next subsection.

\subsubsection{Model for a Fixed Number of VCs}

Because it is difficult to analyze the traffic model taking arrivals of $\mathrm{VC}$ setup requests and releases into account strictly, we introduce a model for a fixed number of VCis in order to simplify the analysis of the traffic characteristics on the VP shortly after a $V \mathrm{C}$ : setup request arrives (Fig.3). In this model we assume that a $\mathrm{VC}$ connection setup request arrives if and only if the same class of VC: connection has just been released. Our parameter design method can be divided into two parts: first, deciding the appropriate smoothing parameter in special cases (i.e., for a fixed number of established VC's in the VP); and second, extending this general case by taking the weighted mean of these smoothing parameters.

The model for a fixed Number of VC's is based on the following assumptions. 
- As soon as a VC: connection is released, a VC: connection of the same class arrives and is accepted.

- The arrival stream of class- $j \mathrm{VC}$ connections is a Poisson process with arrival rate $\lambda_{j}$. And we assume that there is at most one arrival or one release of $\mathrm{VC}$. connections during one update period, and that judgement of connection acceptance is carried out at the end point of the update period when the connection request has arrived.

- The maximum number of cells arriving from a class- $j \mathrm{VC}$ is $R_{j}$ and the average number is $A_{j}$.

- The cell arrival process from an individual VC: has on-states and off-states, and cells are generated only during on-states according to its peak cell rate. In addition, state transitions between an on-state and an off-state occur only at the border of two consecutive measurement periods. Consider the length of an on-state $X_{j}$ and the length of an off-state $Y_{j}$. Let $L_{j}$ be the average length of an on-state. The probability distributions of $X_{j}$ and $Y_{j}$ are given by

$$
\operatorname{Pr}\left\{X_{j}=k w\right\}=\left(1-p_{j}\right) p_{j}^{k-1}, \operatorname{Pr}\left\{Y_{j}=k: v\right\}=\left(1-q_{j}\right) q_{j}^{k-1} k \geq 1,
$$

where $p_{j}$ and $q_{j}$ are given by

$$
p_{j}=1-\frac{w}{L_{j}}, q_{j}=1-\frac{A_{j} w}{L_{j}\left(R_{j}-A_{j}\right)} .
$$

Now we introduce the following notations and assumptions.

\subsubsection{Notation}

$R_{T}: R_{T}=\sum_{j=1}^{m} n_{j} R_{j}$

$\lambda_{T}^{-1}: \lambda_{T}=\sum_{j=1}^{m} n_{j} \lambda_{j}$

$N$ : the number of measurement periods that make up one update period

$\{\Psi(t)=j\}$ : the event of a class- $j$ VC connection being admitted during the th update period.

$\left.X\right|_{A}$ : the conditional probability of random variable $X$ under the condition that event A has occurred.

$M_{j}$ : a random variable expressing the number of $\mathrm{VC}$ connections in on-states under the condition that $n_{j}$ VC. connections exist in the VP.

$\boldsymbol{\pi}^{(i)}={ }^{T}\left(\pi^{(i)}(0) . \pi^{(i)}(1), \cdots\right)$ : stationary probability distribution of random variable $M_{j}$. That is

$$
\boldsymbol{\pi}^{(j)}=\left(\begin{array}{c}
\operatorname{Pr}\left\{M_{j}=0\right\} \\
\operatorname{Pr}\left\{M_{j}=1\right\} \\
\vdots \\
\operatorname{Pr}\left\{M_{j}=n_{j}\right\}
\end{array}\right) .
$$

It is easiy to see that this is a binomial distribution with parameter $n_{j}, A_{j} / R_{j}$.

$\Theta^{(j)}:\left(n_{j} R_{j}+1\right) \times\left(n_{j}+1\right)$ : matrix whose $(i+1, x+1)$ component is $\delta_{i, x} R_{j}, i=$ $0, \cdots, n_{j} R_{j}, x=0, \cdots, n_{j}$, where $\delta_{i, j}$ is Kronecker's symbol. 


\subsubsection{Assumptions}

Assume that there are the following limits on random vectors under the Fixed Number of VC.s Model. We write $q(k: \mathbf{n}), \hat{B}^{(j)}(\mathbf{n})$ as $q(k), \hat{B}^{(j)}$ for simplicity.

$\mathbf{q}={ }^{T}(q(0), q(1), \cdots)$ : random vector which means the measured frequency distribution during a fixed-length interval $w$ for a stationary state. That is

$$
\mathbf{q} \sim_{d} \lim _{t \rightarrow \infty} \frac{1}{t} \sum_{s=1}^{t} \mathbf{q}(s)
$$

$\hat{\mathbf{p}}_{-}^{(j)}={ }^{T}\left\{\hat{p}_{-}^{(j)}(0), \hat{p}_{-}^{(j)}(1), \cdots\right\}$ : limit of the random variable $\hat{\mathbf{p}}_{-}^{(j)}(t)$ just before a class- $j$ VC connection is admitted.

$$
\hat{\mathbf{p}}_{-}^{(j)}=\left.\lim _{t \rightarrow \infty} \hat{\mathbf{p}}(t-1)\right|_{\Psi(t)=j}
$$

$\hat{\mathbf{p}}_{+}^{(j)}={ }^{T}\left\{\hat{p}_{+}^{(j)}(0), \hat{p}_{+}^{(j)}(1), \cdots\right\}$ : limit of the random variable $\hat{\mathbf{p}}_{+}^{(j)}(t)$ just after a class- $j V \mathrm{C}$ : connection is admitted.

$$
\hat{\mathbf{p}}_{+}^{(j)}=\lim _{t \rightarrow \infty} \Upsilon_{j}\left[\left.\hat{\mathbf{p}}(t)\right|_{\Psi(t)=j}\right],
$$

where, for $\hat{\mathbf{p}}(t)={ }^{T}(\hat{p}(0: t), \hat{p}(1: t), \cdots)$,

$$
\Upsilon_{j}[\hat{\mathbf{p}}(t)]=\Phi_{j}\left[\Omega_{j}[\hat{\mathbf{p}}(t)]\right]=\left(\begin{array}{c|c}
0 \\
\vdots \\
0 & 1 \\
\hat{p}(0: t) & R_{T_{j}} \\
R_{j}+1 \\
\vdots \\
\hat{p}_{T}\left(R_{T}-R_{j}-1: t\right) \\
\sum_{k=R_{T}-R_{j}}^{\infty} \hat{p}(k: t) \\
0 \\
\vdots
\end{array}\right)
$$

\subsection{Percentile Point of Distribution $\hat{B}^{(i)}(\mathbf{n})$}

We analyze the relationship between the smoothing parameter and a percentile point of the distribution of random variable $\hat{B}^{(j)}(\mathbf{n})$. The parameter design method in this paper is based on this analysis. We write the expectation of a random variable $X$ as $E[X]$.

Now we define the average and the autocovariance matrix of the random variable $\hat{\mathbf{p}}_{+}^{(j)}$ by the following.

$$
\begin{aligned}
E\left[\hat{\mathbf{p}}_{+}^{(j)}\right] & ={ }^{T}\left(E\left[\hat{p}_{+}^{(j)}(0)\right], E\left[\hat{p}_{+}^{(j)}(1)\right], \cdots\right) \\
\operatorname{Cov}\left\{\hat{\mathbf{p}}_{+}^{(j)}, \hat{\mathbf{p}}_{+}^{(j)}\right\} & =E\left[{ }^{T} \hat{\mathbf{p}}_{+}^{(j)} \cdot \hat{\mathbf{p}}_{+}^{(j)}\right]-{ }^{T} E\left[\hat{\mathbf{p}}_{+}^{(j)}\right] E\left[\hat{\mathbf{p}}_{+}^{(j)}\right] .
\end{aligned}
$$

Using this. the estimate $\hat{B}^{(j)}$ is represented by

$$
\hat{B}^{(j)}=\frac{\boldsymbol{\vartheta}_{r} \cdot \hat{\mathbf{p}}_{+}^{(j)}}{\boldsymbol{\vartheta}_{0} \cdot \hat{\mathbf{p}}_{+}^{(i)}}
$$


Here $\boldsymbol{\vartheta}_{r}=\{\overbrace{0, \cdots, 0}^{r+1}, 1,2, \cdots\}, \boldsymbol{\vartheta}_{0}=\{0,1,2,3, \cdots\}$.

Next, we use the asymptotic expansion shown in [10] to calculate approximately the average and the variance of $\hat{B}^{(i)}$.

$$
\begin{aligned}
& E\left[\hat{B}^{(j)}\right] \\
& \simeq \frac{\boldsymbol{\vartheta}_{r} \cdot E\left[\hat{\mathbf{p}}_{+}^{(j)}\right]}{\boldsymbol{\vartheta}_{0} \cdot E\left[\hat{\mathbf{p}}_{+}^{(j)}\right]}+\frac{\boldsymbol{\vartheta}_{r} \cdot E\left[\hat{\mathbf{p}}_{+}^{(j)}\right]}{\left\{\boldsymbol{\vartheta}_{0} \cdot E\left[\hat{\mathbf{p}}_{+}^{(j)}\right]\right\}^{3}} \cdot \boldsymbol{\vartheta}_{0} \cdot \operatorname{Cov}\left\{\hat{\mathbf{p}}_{+}^{(j)}, \hat{\mathbf{p}}_{+}^{(j)}\right\} \cdot{ }^{T} \boldsymbol{\vartheta}_{0}-\frac{\boldsymbol{\vartheta}_{r} \cdot \operatorname{Cov}\left\{\hat{\mathbf{p}}_{+}^{(j)} \cdot \hat{\mathbf{p}}_{+}^{(j)}\right\} \cdot{ }^{T} \boldsymbol{\vartheta}_{0}}{\left\{\boldsymbol{\vartheta}_{0} \cdot E\left[\hat{\mathbf{p}}_{+}^{(j)}\right]\right\}^{2}} \\
& \operatorname{Var}\left[\hat{B}^{(j)}\right] \\
& \simeq\left\{\frac{\boldsymbol{\vartheta}_{r} \cdot E\left[\hat{\mathbf{p}}_{+}^{(j)}\right]}{\boldsymbol{\vartheta}_{0} \cdot E \cdot\left[\hat{\mathbf{p}}_{+}^{(j)}\right]}\right\}^{2} \cdot\left\{\frac{\boldsymbol{\vartheta}_{r} \cdot \operatorname{Cov}\left\{\hat{\mathbf{p}}_{+}^{(j)}, \hat{\mathbf{p}}_{+}^{(j)}\right\} \cdot{ }^{T} \boldsymbol{\vartheta}_{0}}{\left\{\boldsymbol{\vartheta}_{r} \cdot E\left[\hat{\mathbf{p}}_{+}^{(j)}\right]\right\}^{2}}+\frac{\boldsymbol{\vartheta}_{0} \cdot \operatorname{Cov}\left\{\hat{\mathbf{p}}_{+}^{(j)}, \hat{\mathbf{p}}_{+}^{(j)}\right\} \cdot{ }^{T} \boldsymbol{\vartheta}_{0}}{\left\{\boldsymbol{\vartheta}_{0} \cdot E\left[\hat{\mathbf{p}}_{+}^{(j)}\right]\right\}^{2}}\right. \\
& \left.-\frac{2 \boldsymbol{\vartheta}_{r} \cdot \operatorname{Cov}\left\{\hat{\mathbf{p}}_{+}^{(j)}, \hat{\mathbf{p}}_{+}^{(j)}\right\} \cdot{ }^{T} \boldsymbol{\vartheta}_{0}}{\boldsymbol{\vartheta}_{r} \cdot E\left[\hat{\mathbf{p}}_{+}^{(j)}\right] \cdot \boldsymbol{\vartheta}_{0} \cdot E\left[\hat{\mathbf{p}}_{+}^{(j)}\right]}\right\} .
\end{aligned}
$$

Here we remark that the vector $E\left[\hat{\mathbf{p}}_{+}^{(j)}\right]$ and the matrix $\operatorname{Cov}\left\{\hat{\mathbf{p}}_{+}^{(j)}, \hat{\mathbf{p}}_{+}^{(j)}\right\}$ can be calculated numerically using the following theorem.

Theorem 2.1E $E\left[\hat{\mathbf{p}}_{+}^{(j)}\right]$ and $\operatorname{Cov}\left\{\hat{\mathbf{p}}_{+}^{(j)}, \hat{\mathbf{p}}_{+}^{(j)}\right\}$ satisfy the follouing simultaneous equations,

$$
\begin{aligned}
E\left[\hat{\mathbf{p}}_{+}^{(j)}\right]= & \sum_{i=1}^{m} \frac{n_{i} \lambda_{i}}{\lambda_{T}} \cdot \frac{1-\epsilon^{-\lambda_{T} N_{w}}}{1-(1-\alpha) \epsilon^{-\lambda_{T} N^{2} w}} \cdot \Upsilon_{j}\left[E\left[\hat{\mathbf{p}}_{+}^{(i)}\right]\right] \\
& +\frac{\alpha \epsilon^{-\lambda_{T} N u}}{1-(1-\alpha) \epsilon^{-\lambda_{T} N w}} \Upsilon_{j}[E[\mathbf{q}]] \\
\operatorname{Cov}\left\{\hat{\mathbf{p}}_{+}^{(j)}, \hat{\mathbf{p}}_{+}^{(j)}\right\}= & \sum_{i=1}^{m} \frac{n_{i} \lambda_{i}}{\lambda_{T}} \cdot \frac{1-\epsilon^{-\lambda_{T} N w}}{1-(1-\alpha)^{2} \epsilon^{-\lambda_{T} N_{w} w}} \cdot \Upsilon_{j}\left[\operatorname{Cov}\left\{\hat{\mathbf{p}}_{+}^{(i)}, \hat{\mathbf{p}}_{+}^{(i)}\right\}\right] \\
& +\sum_{s=2}^{\infty} E\left[\alpha^{2} \sum_{k, l=0}^{s-2}(1-\alpha)^{2 s-4-k-l} \cdot \Upsilon_{j}[\operatorname{Cov}\{\mathbf{q}(k), \mathbf{q}(l)\}] \mid s\right] d p(s)
\end{aligned}
$$

In particular, if the average length of an on-state is infinite under the condition that other parameters of the model are fixed, in other woords,

$$
L_{j} \rightarrow \infty, \text { for } j=1,2, \cdots, m,
$$

(This is same with the fact that $p_{j}, q_{j}-\infty$ in section 2.5.3.) then the second equation is written in the following form

$$
\begin{aligned}
\operatorname{Cov}\left\{\hat{\mathbf{p}}_{+}^{(j)}, \hat{\mathbf{p}}_{+}^{(j)}\right\}= & \sum_{i=1}^{m} \frac{n_{i} \lambda_{i}}{\lambda_{T}} \cdot \frac{1-\epsilon^{-\lambda_{T} N w}}{1-(1-\alpha)^{2} \epsilon^{-\lambda_{T} N_{w}}} \cdot \Upsilon_{j}\left[\operatorname{Cov}\left\{\hat{\mathbf{p}}_{+}^{(i)}, \hat{\mathbf{p}}_{+}^{(i)}\right\}\right] \\
& +\Upsilon_{j}\left[\frac{\alpha^{2}}{1-(1-\alpha)^{2} \epsilon^{-\lambda_{T} N_{w}}}\left\{\epsilon^{-\lambda_{T} N w}+\frac{2(1-\alpha) \epsilon^{-2 \lambda_{T} N w}}{1-(1-\alpha) \epsilon^{-\lambda_{T} N_{w}}}\right\} \phi\right]
\end{aligned}
$$

where the coefficient $E[\mathbf{q}], \phi$ is given by 


$$
\begin{aligned}
E[\mathbf{q}] & =\left\{\Theta^{(1)} \cdot \boldsymbol{\pi}^{(1)}\right\} \star \cdots \star\left\{\Theta^{(m)} \cdot \boldsymbol{\pi}^{(m)}\right\} \\
\phi & =\operatorname{diag}\{E[\mathbf{q}]\}-E[\mathbf{q}] \cdot{ }^{T} E[\mathbf{q}]
\end{aligned}
$$

and the difnition of symbol $\star$ is given in appendix.

Note that the first equation is invariant for $L_{j}-\infty$ because $E[q]$ is independent from $L_{j}$. Proof. See appendix A.

When $L_{j}-\infty$, where $j=1, \cdots, m$, the $b \%$ percentile of $\hat{B}^{(j)}$ takes the smallest value (see appendix $B$ ). In this case, the probability that a new VC connection setup request is admitted, derived from this, becomes larger. Now we wish to limit the error probability that a new VC: is admitted when the VP has been saturated with $V C$ s, so the assumption that $L_{j} \rightarrow \infty,(j=1,2, \cdots . m)$ is appropriate for designing a smoothing parameter.

We use notations $\tilde{\mathbf{p}}_{+}^{(j)}$ and $\tilde{B}^{(j)}$ in place of $\hat{\mathbf{p}}_{+}^{(j)}$ and $\hat{B}^{(j)}$, when $L_{j}-\infty$ with $j=1, \cdots, m$. To estimate the $b \%$ percentile point of the distribution $\hat{B}^{(j)}$ using the average $E\left[\hat{B}^{(j)}\right]$ and the variance $\operatorname{Var}\left[\hat{B}^{(j)}\right]$, we assume that the random variable $\hat{B}^{(j)}$ obeys a lognormal distribution for simplicity. By considering the following relationship (see appendix B)

$$
E\left[\hat{B}^{(j)}\right] \simeq E\left[\tilde{B}^{(j)}\right], \quad \operatorname{Var}\left[\hat{B}^{(j)}\right] \leq \operatorname{Var}\left[\tilde{B}^{(j)}\right],
$$

we can easily see that its $b \%$ percentile point is larger than

$$
\exp \left\{\ln E\left[\tilde{B}^{(j)}\right]-\frac{1}{2} \ln \left\{\frac{\operatorname{Var}\left[\tilde{B}^{(j)}\right]}{E\left[\tilde{B}^{(j)}\right]^{2}}+1\right\}+\alpha_{b} \sqrt{\ln \left\{\frac{\operatorname{Var}\left[\tilde{B}^{(j)}\right]}{E\left[\tilde{B}^{(j)}\right]^{2}}+1\right\}}\right\},
$$

where $\alpha_{b}$ is the $b \%$ percentile point of the standard normal distribution $N(0,1)$.

Consequently, we can decide the most suitable smoothing parameter, written by $\alpha^{(j)}(\mathbf{n})$, i.e., the largest value that satisfies the condition that the $b \%$ percentile of the distribution $\hat{B}^{(j)}(\mathbf{n})$ is below the cell-loss-rate objective $B_{0}$ (Fig.5). This implies that we can expect the advantages of statistical multiplexing while keeping the error probability to below the $b \%$ level.

\subsection{Weighted mean of smoothing parameters}

In the previous section we decided the appropriate smoothing parameters $\left\{a^{(j)}(\mathbf{n})\right\}$ for $\mathbf{n} \in V_{j},(j=1,2, \cdots, m)$ under the assumptions of the Fixed Number of $V C$ 's Model. In this section we will finally decide the most suitable smoothing parameter taking the increases and decreases of the number of established VCs into account. Therefore, we consider the occurence probability of each established class of VC connections $\mathbf{n}$. And to consider the arrivals and departures of $V C$ : connections as the actual case, we take the weighted mean of the smoothing parameters according to the occurence probability.

Now we consider them on the following situation approximately. That is to say, the arrival stream of class- $j \mathrm{VC}$ connection setup requests is a Poisson process with arrival rate $\lambda_{j}$ that holding time is exponential with parameter $\mu_{j}$. Therefore we approximate the occurrence probability $\operatorname{Pr}\{\mathbf{n}\}$ of $\mathbf{n}$ to the following product form, 


$$
\operatorname{Pr}\left\{\mathbf{n}=\left(n_{1}, \cdots, n_{m}\right)\right\}=\frac{\prod_{j=1}^{m} \rho_{j}^{n_{j}}}{\prod_{j=1}^{m} n_{j} !} \exp \left\{-\sum_{j=1}^{m} \rho_{j}\right\}
$$

where $\rho_{j}=\lambda_{j} / \mu_{j}$.

Next, we consider the conditional probability that the next arrival of VC: setup request is of class- $j$ under the condition that the number of established VCs is $\mathbf{n}$. It is easy to see that its conditional probability is $\frac{\lambda_{j}}{\sum_{j=1}^{m} \lambda_{j}}$.

Finally we take the weighted mean of the smoothing parameter $\left\{\alpha^{(j)}(\mathbf{n})\right\}$ as follows,

$$
\alpha=C \cdot \sum_{j} \sum_{\left(n_{1}, \cdots, n_{m}\right) \in V_{j}^{r}} \frac{\prod_{j=1}^{m} \rho_{j}^{n_{j}}}{\prod_{j=1}^{m} n_{j} !} \cdot \frac{\lambda_{j}}{\sum_{j=1}^{m} \lambda_{j}} \alpha^{(j)}\left(n_{1}, \cdots, n_{m}\right),
$$

where $C$ is a normalization constant, given by

$$
C^{-1}=\sum_{j} \sum_{\left(n_{1}, \cdots, n_{m}\right) \in V_{j}} \frac{\prod_{j=1}^{m} \rho_{j}^{n_{j}}}{\prod_{j=1}^{m} n_{j} !} \cdot \frac{\lambda_{j}}{\sum_{j=1}^{m} \lambda_{j}} .
$$

\section{Numerical Examples}

Here, we give some numerical examples to explain our parameter design method. We assume that the VP bandwidth is $150 \mathrm{Mb} / \mathrm{s}$, the length of a measurement period $w$ is $0.36 \mathrm{~ms}(r=128)$, and the number $N$ of measurement periods in an update period is 1000 .

\section{Example 1}

The first example shows the influence of the holding time on $\alpha$. Assume that the cell loss ratio objective $B_{0}$ is $2.0 \times 10^{-5}$ and that there are two call classes. Class- 1 calls arrive at a traffic intensity of $10^{-3}$ calls/second, their mean holding time is $2.50 \mathrm{~s}$, the peak bit rate is $5 \mathrm{Mb} / \mathrm{s}$, and the average bit rate is $1 \mathrm{Mb} / \mathrm{s}$. Class-2 calls arrive at a traffic intensity of $10^{-2}$ calls/second, their mean holding time is $2.5 \mathrm{~s}$, the peak bit rate is $5 \mathrm{Mb} / \mathrm{s}$, and the average bit rate is $1 \mathrm{Mb} / \mathrm{s}$. The region that can admit VCs guaranteeing the cell loss ratio $B_{0}$ is denoted as $V$ (defined in 2.5.1) in Figure $6 . V_{1}$ and $V_{2}$ derived from Eq.(2) are on the line conecting point $(0,100)$ and $(100,0)$.

Consider that a class- $j, j=1,2$ call arrives and requests admission. Under the condition that the number of established $V C$ s is $\mathbf{n}\left(\in V_{1}\right.$ or $\left.V_{2}\right)$, the cell loss ratio estimates are plotted as functions of the parameter $\alpha$ in Figure 7 . Precisely speaking, Figure 7 shows the relationship between $\alpha$ and the $5 \%$ percentile of $\hat{B}^{(j)}(\mathbf{n})$. For example, if there are no class- 2 VCs and there are 100 class- $1 \mathrm{VC}$ s, the relationship between $\alpha$ and the $5 \%$ pecentile of $\hat{B}^{(j)}(\mathbf{n})$ is shown by the curve labelled $(100.0)$ in Figure 7 . If we set $\alpha$ such that the $5 \%$ percentile is equal to the cell loss ratio objective $B_{0}$, we obtain $\alpha^{(1)}(100,0)=0.05$. This implies that if we set $\alpha$ to 0.05 , an arriving class $1 \mathrm{VC}$ is admitted with probability 0.05 when the numbers of established class- $1 \mathrm{VCs}$ and class- $2 \mathrm{VC}$ s are 100 and 0 , respectively. Similarly, $\alpha^{(2)}(0,100)=0.5$. These examples reveal an important property. When the holding time is short, the impact of the peak of each $V C$ has a large impact because there 
are many arrivals, and the probability estimate is often updated by arrivals. As a result, a large $\alpha$ can be used for a fixed cell loss ratio objective, compared with long holding-time cases. In addition, we can find that the parameter $\alpha$ derived from Eq.(8) is 0.26 , which is a value between $\alpha^{(1)}(100,0)$ and $\alpha^{(2)}(0,100)$.

\section{Example 2}

This example investigates the influence of the peak and average cell rates on $\alpha$ when the mean holding times of both classes are the same. Assume that the cell loss ratio objective $B_{0}$ is $6.8 \times 10^{-5}$ and that there are two call classes. Class- 1 calls arrive at the traffic intensity of $10^{-2}$ calls/second, their mean holding time is $25 \mathrm{~s}$, the peak bit rate is 9 $\mathrm{Mb} / \mathrm{s}$, and the average bit rate is $1 \mathrm{Mb} / \mathrm{s}$. Class-2 calls arrive at the traffic intensity $10^{-2}$ calls/second, their peak bit rate is $2.4 \mathrm{Mb} / \mathrm{s}$, the average bit rate is $1.2 \mathrm{Mb} / \mathrm{s}$, and the mean holding time is also $25 \mathrm{~s}$. The region where a new $\mathrm{VC}$ can be admitted is Figure 8. The $a$ derived from Eq.( 8 ) is 0.41 (Figure 9). The ratio of the peak bit rate to the average bit rate is fairly insensitive to $\alpha$.

\section{Example 3}

This example is given under the condition that the average cell rates of both classes are the same. Assume that the cell loss ratio objective $B_{0}$ is $1.0 \times 10^{-6}$ and that there are two call classes. The peak bit rate of class 1 calls is $12 \mathrm{Mb} / \mathrm{s}$ and the average bit rate is $0.6 \mathrm{Mb} / \mathrm{s}$. The peak bit rate of class 2 calls is $1.2 \mathrm{Mb} / \mathrm{s}$ and the average bit rate is $0.6 \mathrm{Mb} / \mathrm{s}$. We consider two cases: (1) Calls of both classes arrive at a traffic intensity $10^{-3}$ calls/second. The mean holding times of both classes are $250 \mathrm{~s}$. (2)Both classes calls arrive at the traffic intensity $4.2 \times 10^{-2}$ calls/second and the mean holding times of both classes are $100 \mathrm{~s}$. Figure 10 illustrates the region $V$. We conclude that the mean holding time is more sensitive to the design of parameter $\alpha$ than is the ratio of the peak bit rate to the average bit rate.

\section{Example 4}

In this example, we assume that the VP bandwidth is $200 \mathrm{Mb} / \mathrm{s}$ and the other conditions are the same as in Example 1, to investigate the influence of the VP bandwidth on $\alpha$. Figure 13 shows the relationship between the cell loss ratio estimate and the smoothing parameter $\alpha$. Compared with Example 1, more VCis can be admitted because the VP bandwidth is higher (Figure 12). The smoothing parameter $\alpha$ derived from Eq. ( 8 ) is 0.35 in this example, which is larger than in Example 1. If we used the value in Example 1 with a VP bandwidth of $200 \mathrm{Mrb} / \mathrm{s}$, the number of the VC that could be admitted would be smaller and the cell loss ratio performance would be better.

\section{Simulation}

Because of the advantage of being quick at figures, it is expected that analytic approach is powerful to estimate cell loss ratio. But, remark that we used some assumptions with respect to the traffic model and the distribution form of $\hat{B}$ in order to analyze percentile 
point of the distribution of $\hat{B}$ simply. Figure 14 and 15 showed simulation results of the frequency distributions of the cell-loss-ratio estimates $\hat{B}$ just before the arrival of the $V C$ setup request. Solid line is the lognormal distribution whose mean and variance is derived as the same manner of section 2. Although the statistics such as the mean and the variance of the estimate $\hat{B}$ derived from the analytic approach are not so different from the simulation results, there is room for improvement of the accuracy. For example, here the distribution of $\hat{B}$ is assumed to be a lognormal distribution in order to analyze its percentile point easy. Other type distributions whose tail are larger than lognormal type distributions may be much better to approximate the distribution of $\hat{B}$. But in general it isn't easy to identify the distribution from two parameters (i.e. mean and variance). It remains for further study.

\section{Conclusion}

This paper developed a method for designing a smoothing parameter for heterogeneous traffic conditions. We consider the error probability that a new $V C$ is admitted when the $V P$ is saturated with VCs, and found that this design method limits the error probability to below a certain fixed level. The main part of the analysis in this paper is the relationship between the smoothing parameter $\alpha$ and the $b \%$ percentile of the estimate of the cell loss ratio upper bound $\hat{B}$. In the analysis, we assumed that a cell arrival process from an individual VC: has on-states and off-states and that cells are generated only during onstates. In order to make the analysis tractable. we introduced the fixed number of $V \mathrm{C} s$ model, whose parameters are the arrival rate of VC:s, the peak cell rate of a VC. Finally we took the weighted sum of the smoothing parameters derived using the fixed number of VC's model.

For future study, a parameter design method using a transient analysis is required.

We have not mentioned how to choose $v$ and $N$. In practice, these parameters are decided from how many $V C$ setup requests arrive during one update period length $v N$. If this number is too large, traffic measurement in each update period cannot be used correctly. Therefore, $u N N$ must be short enough that this number is sufficiently small. When $v N$ is fixed, the balance between $w$ and $N$ can be set by implementation conditions.

\section{A Supplement to Theorem 2.1}

In this appendix, we derive the simultaneous equations of Theorem 2.1.

\section{A.1 Simultaneous Equations of $E\left[\hat{\mathbf{p}}_{+}^{(j)}\right]$}

Suppose that a class- $i$ call arrives at the $t$ th update period, and no more VCis arrives or depart until a class- $j$ call arrives at the $t+s$ th update period. Then the estimated distribution $\hat{\mathbf{p}}_{+}^{(j)}$ of the number of arriving cells follows,

$$
\begin{aligned}
\hat{\mathbf{p}}(t) & =\Upsilon_{i}[\hat{\mathbf{p}}(t-1)] \\
\hat{\mathbf{p}}(t+s-1) & =(1-\alpha)^{s-1} \hat{\mathbf{p}}(t)+\alpha \sum_{h=0}^{s-2}(1-\alpha)^{h} \mathbf{q}(t+s-2-h)
\end{aligned}
$$


Now, let the time-origin be the beginning of the 1st update period, and let $d p(s)$ be the probability that a call arrives during the sth update period. By considering the probability under the condition that a class- $i$ call arrives during the $t$ th update period, we obtain

$$
\begin{aligned}
& \sum_{s=1}^{\infty} E[\hat{\mathbf{p}}(t+s-1) \mid \Psi(t+s)=j, s] d p(s) \\
& \quad=\sum_{i=1}^{m} \sum_{s=1}^{\infty} E[\hat{\mathbf{p}}(t+s-1) \mid \Psi(t)=i, \Psi(t+s)=j, s] d p(s) \cdot \frac{n_{i} \lambda_{i}}{\lambda_{T}} r \\
& \quad=\sum_{i=1}^{m} \sum_{s=1}^{\infty} E\left[(1-\alpha)^{s-1} \Upsilon_{i}[\hat{\mathbf{p}}(t-1)]+\alpha \sum_{h=0}^{s-2}(1-\alpha)^{h} \mathbf{q}(t+s-2-h) \mid s\right] d p(s) \cdot \frac{n_{i} \lambda_{i}}{\lambda_{T}} \\
& \quad=\sum_{i=1}^{m} \frac{n_{i} \lambda_{i}}{\lambda_{T}} \sum_{s=1}^{\infty} E\left[(1-\alpha)^{s-1} \mid s\right] \cdot\left(\Upsilon_{i}[E[\hat{\mathbf{p}}(t-1) \mid \Psi(t)=i]]-E[\mathbf{q}]\right) d p(s)+E[\mathbf{q}] .
\end{aligned}
$$

Since

$$
\sum_{s=1}^{\infty} E\left[(1-\alpha)^{s-1} \mid s\right] d p(s)=\sum_{s=1}^{\infty} \int_{(s-1) N_{w}}^{s N w} \lambda_{T} \epsilon^{-\lambda_{T} w t} d t=\frac{1-\epsilon^{-\lambda_{T} N_{w}}}{1-(1-\alpha) \epsilon^{-\lambda_{T} N^{*} w}}
$$

leting $t$ tend to infinity, we get

$$
E\left[\hat{\mathbf{p}}_{-}^{(j)}\right]=\sum_{i=1}^{m} \frac{n_{i} \lambda_{i}}{\lambda_{T}} \cdot \frac{1-\epsilon^{-\lambda_{T} N w}}{1-(1-\alpha) \epsilon^{-\lambda_{T} N_{w}}}\left\{\Upsilon_{i}\left[E\left[\hat{\mathbf{p}}_{-}^{(i)}\right]\right]-E[\mathbf{q}]\right\}+E[\mathbf{q}],
$$

or

$$
E\left[\hat{\mathbf{p}}_{+}^{(i)}\right]=\sum_{i=1}^{m} \frac{n_{i} \lambda_{i}}{\lambda_{T}} \cdot \frac{1-\epsilon^{-\lambda_{T} N_{w}}}{1-(1-\alpha) \epsilon^{-\lambda_{T} N_{w}}}\left\{\Upsilon_{j}\left[E\left[\hat{\mathbf{p}}_{+}^{(i)}\right]\right]-\Upsilon_{j}[E[\mathbf{q}]]\right\}+\Upsilon_{j}[E[\mathbf{q}]]
$$

\section{A.2 Simultaneous equations of $\operatorname{Cov}\left\{\hat{\mathbf{p}}_{+}^{(j)}, \hat{\mathbf{p}}_{+}^{(j)}\right\}$}

Now assume

$$
\operatorname{Cov}\left\{\hat{\mathbf{p}}(t), \sum_{h=0}^{s-2}(1-\alpha)^{h} \mathbf{q}(t+s-2-h) \mid \Psi(t)=i, \Psi(t+s)=j, s\right\}=0 .
$$

This means that we consider only the correlation of the measured cells arriving from the th update period to the $t+m$ th update period, and we can neglect the effect of cells that arrive before the $t$ th update period. This is true when cells arrival intervals are long enough. Therefore, by the same manner as appendix A.1, we get the result.

\section{A.3 $E[q]$ and $\phi$ in the coefficients of Theorem 2.1}

Here we will express $E[\mathbf{q}]$ and $\phi$ in the coefficients of Theorem 2.1 by $\Theta^{(j)}$ and $\pi^{(j)}$ $(j=1,2, \cdots, m)$. 


\section{A.3.1 Expanded expression of $E[\mathbf{q}]$}

First we introduce a convolution for two matrices $A$ and $B: A=\left(a_{i, j}\right)$ and $B=\left(b_{i, j}\right)$. That is,

$$
A \star B=\left(\sum_{h_{1}+h_{2}=i, k_{1}+k_{2}=j} a_{h_{1}, k_{1}} \cdot b_{h_{2}, k_{2}}\right) \text {. }
$$

Here, of course. we also use the previous notations. Let $N_{t, k}$ be the number of the measured cells in the $k$ th measurement interval in the $t$ update period. Let $\mathbf{e}_{t, k}=\delta\left(N_{t, k}\right)$, where $(i+1)$ th

$\delta(i)={ }^{T}\{\underbrace{0,0, \cdots, \overbrace{1}^{1}, 0, \cdots}\}$. Then, from the definition of $\mathbf{q}(t)$, we get

$$
E[\mathbf{q}(t)]=\frac{1}{N} \sum_{k=1}^{N} E\left[\mathbf{e}_{t, k}\right]=\frac{1}{N} \sum_{k=1}^{N} \operatorname{Pr}\left\{\mathbf{e}_{t, k}=\delta(i)\right\} .
$$

Observe that

$$
\begin{aligned}
\operatorname{Pr}\left\{\mathbf{e}_{t, k}=\delta(i)\right\} & =\operatorname{Pr}\left\{N_{t, k}=i\right\} \\
& =\sum_{k_{1}+\cdots+k_{m}=i} \operatorname{Pr}\left\{N_{t, k}^{(1)}=k_{1}\right\} \cdots \operatorname{Pr}\left\{N_{t, k}^{(m)}=k_{m}\right\},
\end{aligned}
$$

and that the $\left(k_{j}+1\right)$ th component of a column vector $\Theta^{(j)} \cdot \pi^{(j)}$ is $\operatorname{Pr}\left\{N_{t, k}^{(j)}=k_{j}\right\}$ for $j=1,2, \cdots, m$. Therefore

$$
E[\mathbf{q}(t)]=\left\{\Theta^{(1)} \cdot \boldsymbol{\pi}^{(1)}\right\} \star \cdots \star\left\{\Theta^{(m)} \cdot \boldsymbol{\pi}^{(m)}\right\} \quad(=E[\mathbf{q}]) .
$$

Note that right-hand side of the equation does not depend on $t$ and that $q(k: \mathbf{n})$ in the section 2.5 .1 is equal to $E[q(k, t)]$ for $k=0,1,2, \cdots$.

\section{A.3.2 Expanded Expression of $\operatorname{Cov}\{\mathbf{q}(k), \mathbf{q}(k+h)\}$}

We consider the covariance matrix of the measured frequency distribution $\mathbf{q}(k)$ and $\mathbf{q}(k+$ h). By the definition, we have

$$
\operatorname{Cov}\{\mathbf{q}(k), \mathbf{q}(k+h)\}=\frac{1}{N^{2}} \sum_{k_{1}=1, k_{2}=1}^{N} E\left[\mathbf{e}_{k, k_{1}} \cdot{ }^{T} \mathbf{e}_{k+h, k_{2}}\right]-E[\mathbf{q}(k)] \cdot{ }^{T} E[\mathbf{q}(k+h)] .
$$

Here the $\left(h_{1}+1, h_{2}+1\right)$-component of the matrix $E\left[\mathbf{e}_{k ; k_{1}} \cdot{ }^{T} \mathbf{e}_{k+h, k_{2}}\right]$ is given by

$$
\begin{aligned}
& \operatorname{Pr}\left\{\mathbf{e}_{k, k_{1}}=\delta\left(h_{1}\right), \mathbf{e}_{k+h, k_{2}}=\delta\left(h_{2}\right)\right\} \\
& \quad=\operatorname{Pr}\left\{N_{k, k_{1}}=h_{1}, N_{k+h, k_{2}}=h_{2}\right\} \\
& =\sum_{\mathbf{u}_{1}+\cdots+\mathbf{u}_{\mathrm{m}}=\mathbf{h}_{1}, \mathbf{v}_{2}+\cdots+\mathbf{v}_{\mathrm{m}}=\mathrm{h}_{2}} \operatorname{Pr}\left\{\mathrm{N}_{\mathbf{k}, \mathbf{k}_{1}}^{(1)}=\mathrm{u}_{1}, \mathrm{~N}_{\mathbf{k}+\mathbf{h}, \mathbf{k}_{2}}^{(1)}=\mathrm{v}_{1}\right\} \cdots \operatorname{Pr}\left\{\mathrm{N}_{\mathbf{k}, \mathbf{k}_{1}}^{(\mathrm{m})}=\mathrm{u}_{\mathrm{m}}, \mathrm{N}_{\mathbf{k}+\mathbf{h}, \mathbf{k}_{2}}^{(\mathrm{m})}=\mathrm{v}_{\mathrm{m}}\right\} .
\end{aligned}
$$

Observe that

$$
\operatorname{Pr}\left\{N_{k, k_{1}}^{(j)}=u_{j}, N_{k+h_{, k}}^{(j)}=v_{j}\right\} \leq \operatorname{Pr}\left\{N_{k, k_{1}}^{(j)}=u_{j}\right\},
$$

and that the right-hand side is the $\left(u_{j}+1\right)$ th component of a column vector $\Theta^{(j)} \cdot \pi^{(j)}$ for $j=1,2, \cdots, m$. We have

$$
E\left[\mathbf{e}_{k, k_{1}} \cdot{ }^{T} \mathbf{e}_{k+h, k_{2}}\right] \leq \operatorname{diag}\left\{\left[\Theta^{(1)} \cdot \boldsymbol{\pi}^{(1)}\right] \star \cdots \star\left[\Theta^{(m)} \cdot \boldsymbol{\pi}^{(m)}\right]\right\} \quad(=E[\boldsymbol{q}]),
$$


where $\operatorname{diag}\{A\}$ for a column vector $A$ means the diagonal matrix whose component is same as that of $A$. Therefore

$$
\operatorname{Cov}\{\mathbf{q}(k), \mathbf{q}(k+h)\} \leq \operatorname{diag}\{E[\boldsymbol{q}]\}-\{E[\boldsymbol{q}]\} \cdot{ }^{T}\{E[\boldsymbol{q}]\}
$$

We write the right-hand side of the inequality as $\phi$.

\section{Remark}

If $L_{j} \quad(j=1,2, \cdots, m)$ is infinity, then

$$
\operatorname{Pr}\left\{N_{k, k_{1}}^{(j)}=u_{j}, N_{k+h, k_{2}}^{(j)}=v_{j}\right\}=\operatorname{Pr}\left\{N_{k, k_{1}}^{(j)}=u_{j}\right\}
$$

hold. Therefore the equality hold in Eq.(9).

\section{B When $L_{j} \rightarrow \infty,(j=1,2, \cdots, m)$ is Infinity}

In this section we analyze the $b \%$ percentile of the distribution $\hat{B}^{(j)}$ optimistically. We use notations $\hat{\mathbf{p}}_{+}^{(j)}, \hat{B}^{(j)}, \tilde{\mathbf{p}}_{+}^{(j)}$, and $\tilde{B}^{(j)}$ as section 2.6. Recall that the denominator of Eq.(3) is the sample average of cells arriving during a measurement period and the numerator is that of lost cells. In general in ATM Networks, the cell-loss-ratio is expected to be so small $\left(10^{-6}\right.$ to $\left.10^{-9}\right)$ that the coefficient of variation of the denominator is sufficiently smaller than that of the numerator. Therefore, the following equations hold approximately.

$$
\begin{aligned}
E\left[\hat{B}^{(j)}\right] & \simeq \frac{\boldsymbol{\vartheta}_{r} \cdot E\left[\hat{\mathbf{p}}_{+}^{(j)}\right]}{\boldsymbol{\vartheta}_{0} \cdot E\left[\hat{\mathbf{p}}_{+}^{(j)}\right]} \\
\operatorname{Var}\left[\hat{B}^{(j)}\right] & \simeq \frac{\boldsymbol{\vartheta}_{r} \cdot \operatorname{Cov}\left\{\hat{\mathbf{p}}_{+}^{(j)}, \hat{\mathbf{p}}_{+}^{(j)}\right\} \cdot{ }^{T} \boldsymbol{\vartheta}_{0}}{\left\{\boldsymbol{\vartheta}_{0} \cdot E\left[\hat{\mathbf{p}}_{+}^{(j)}\right]\right\}^{2}} .
\end{aligned}
$$

On other hand, from the structure of Fq.(5), we can see that

$$
E\left[\hat{\mathbf{p}}_{+}^{(j)}\right]=E\left[\tilde{\mathbf{p}}_{+}^{(j)}\right] .
$$

And from the structure of Eqs. (4), (6) and (9),

$$
\operatorname{Cov}\left\{\hat{\mathbf{p}}_{+}^{(j)}, \hat{\mathbf{p}}_{+}^{(j)}\right\} \leq \operatorname{Cov}\left\{\tilde{\mathbf{p}}_{+}^{(j)}, \tilde{\mathbf{p}}_{+}^{(j)}\right\}
$$

Here $\leq$ means that the inequality holds component-wise. Therefore,

$$
\operatorname{Var}\left[\hat{B}^{(j)}\right] \leq \operatorname{Var}\left[\tilde{B}^{(j)}\right] .
$$

Therefore, when $L_{j} \rightarrow \infty$, where $j=1,2, \cdots, m$, then

$$
E\left[\hat{B}^{(j)}\right] \simeq E\left[\tilde{B}^{(j)}\right], \quad \operatorname{Var}\left[\hat{B}^{(j)}\right] \leq \operatorname{Var}\left[\tilde{B}^{(j)}\right] .
$$

This means the $b \%$ percentile of distribution $\tilde{B}^{(j)}$ is smaller than that of $\hat{B}^{(j)}$ assuming that both obey the same type distribution. 


\section{References}

[1] ITU-T Recommendation I.371, "Traffic Control and Congestion Control in B-ISDN," (1992).

[2] Kawamura Y. and Saito H., "Parameter Design of Dynamic Admission Control in ATM Networks," 1993 IEICE Spring Conference, Vol. B-482, 1993 (in Japanese).

[3] Shiomoto K. and Iwata H., "Performance Evaluation of Call Admission Control Using Traffic Measurement in ATM Networks," IEICE National Autumn Conference, Vol. B-293, 1991.

[4] Saito H., "Teletraffic Technologies in ATM Networks," Artech House, Boston (1994).

[5] Chaki S., Saito H., and Miyake K., "Reactive Congestion Control for High SpeedData Communication ATM Networks," IEICE Trans. Commun. Vol.J76-B-I, No.11, pp.838-848, November 1993 (in Japanese).

[6] Fujiki M. and Ganbe E., "Teletraffic Theory," maruzen, 1980 (in Japanese).

[7] Kawamura Y. and Saito H., "A Study of Dynamic Connection Admission Control with Traffic Measurement in ATM Networks," IEICE. Technical Report, CS93-151, IE93-88, pp.45-53, December 1993 (in Japanese).

[8] Saito H., Kawashima K., and Sato K., "Traffic Control Technologies in ATM Networks," IEICE Transactions, E74, 4, pp.761-771(1991).

[9] Saito H. and Shiomoto K., "Dynamic Call Admission Control in ATM Networks," IEEE J. Selected Areas in Communications, 9, 7, pp.982-989(1992).

[10] Saito H., "Hybrid Connection Admission Control in ATM Networks," IEEE Communications Society IEEE International Conference on Communications June 14-18, pp.699-703,(1992).

[11] Saito H., "Call Admission Control using Upper Bound of Cell Loss Probability," IEEE Transactions on Communications, 40, 9, pp.1512-1521(1992).

[12] Kawamura Y. and Saito H., "Parameter Design of Dynamic Admission Control in ATM Networks; Heterogeneous Traffic," 1994 IEICE Spring Conference, Vol. B-523, 1994 (in Japanese).

Yoshinori Kawamura received the B.S. and M.S. degrees in mathematics from Nagoya University, Nagoya, Japan, in 1986 and 1989, respectively.

In 1989, he joined NTT Electrical Communications Laboratories, Tokyo, Japan, where he has worked on traffic engineering in ATM networks.

Hiroshi Saito received the B.E. degree in mathematical engineering, the M.E. degree in control engineering and the Ph.D.degree in teletraffic engineering from the University of Tokyo, Tokyo, Japan, in 1981,1983 and 1992, respectively.

He joined NTT in 1983. He is currently working in the area of teletraffic design, control, and administration of telecommunications networks at the NTT Telecommunication Networks Laboratories, Tokyo, Japan. 


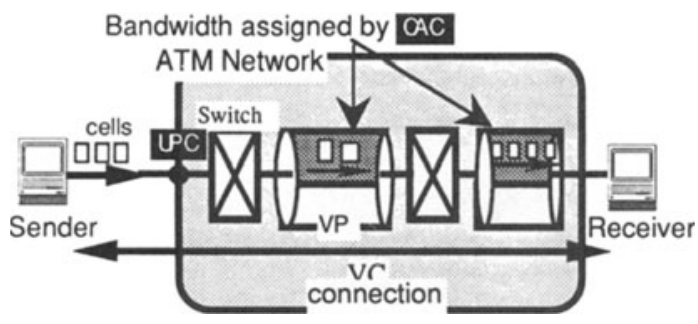

Fig. 1. Connection Admission Control.
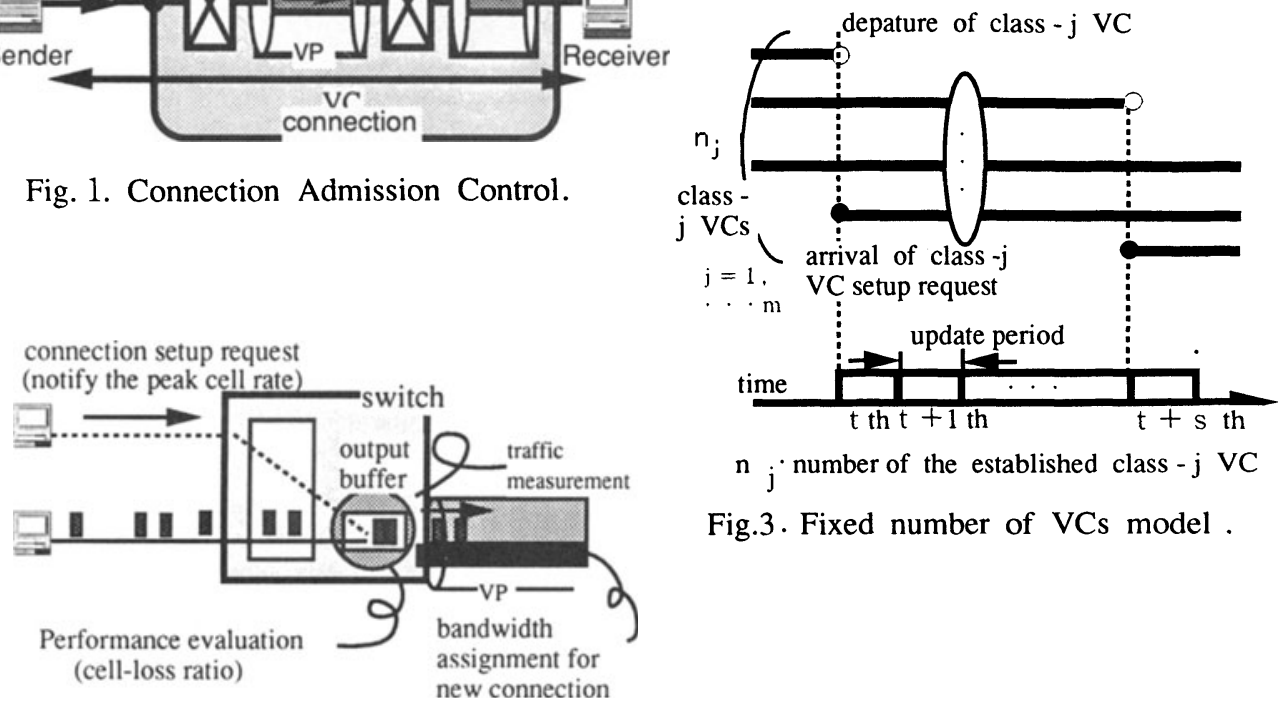

$n_{j} \cdot$ number of the established class $-\mathrm{j}$ VC

Fig.3. Fixed number of VCs model .

Fig. 2. CAC using traffic measurement

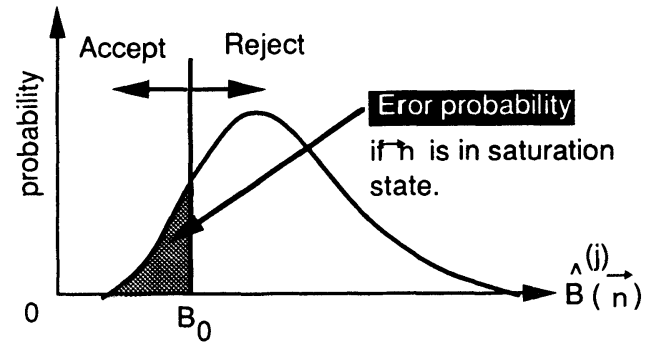

Fig. 4. Error probability

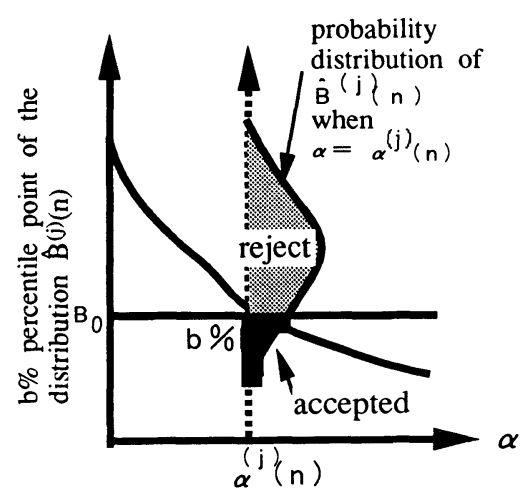

Fig. 5. The $b \%$ percentile point of the estimated cell-loss-ratio distributin $\hat{B}^{(j)}(\mathrm{n})$. 


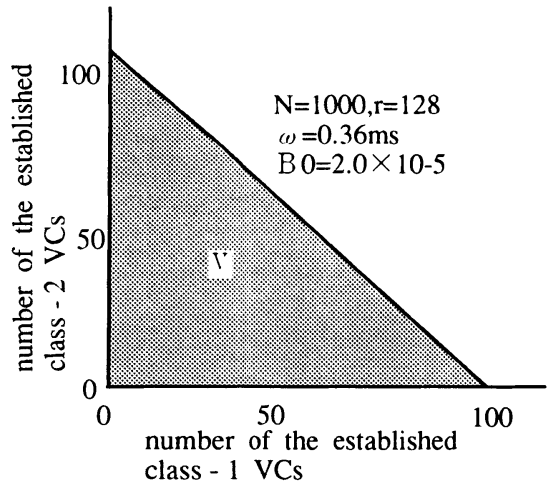

VP bandwidth: $150 \mathrm{Mb} / \mathrm{s}$

class peak cell rate average cell rate

$\begin{array}{lll}1 & 5 \mathrm{Mb} / \mathrm{s} & 1 \mathrm{Mb} / \mathrm{s} \\ 2 & 5 \mathrm{Mb} / \mathrm{s} & 1 \mathrm{Mb} / \mathrm{s}\end{array}$

Fig .6. Region that can admit VCs .

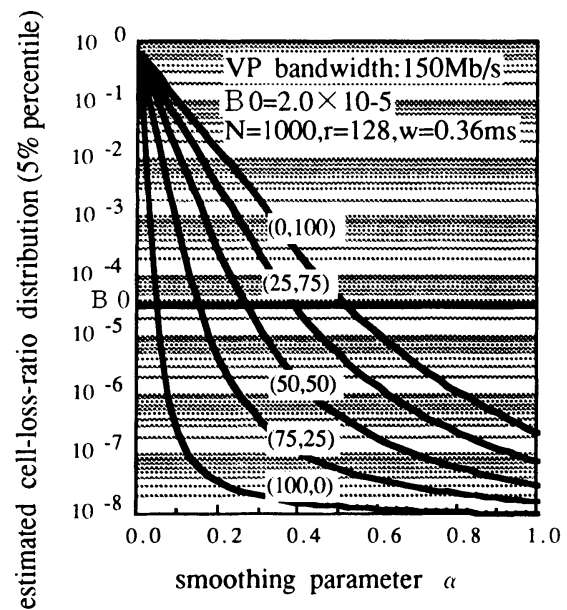

\begin{tabular}{lll}
\multicolumn{1}{c}{ class } & \multicolumn{1}{c}{1} & \multicolumn{1}{c}{2} \\
peak cell rate & $5 \mathrm{Mb} / \mathrm{s}$ & $5 \mathrm{Mb} / \mathrm{s}$ \\
average cell rate & $1 \mathrm{Mb} / \mathrm{s}$ & $1 \mathrm{Mb} / \mathrm{s}$ \\
arrival rate & $10 \mathrm{e}-3 \mathrm{calls} / \mathrm{s}$ & $10 \mathrm{e}-2 \mathrm{calls} / \mathrm{s}$ \\
mean holding time & $250 \mathrm{~s}$ & $25 \mathrm{~s}$
\end{tabular}

Fig. 7. The $5 \%$ percentile point of the estimated cell-loss-ratio distribution.

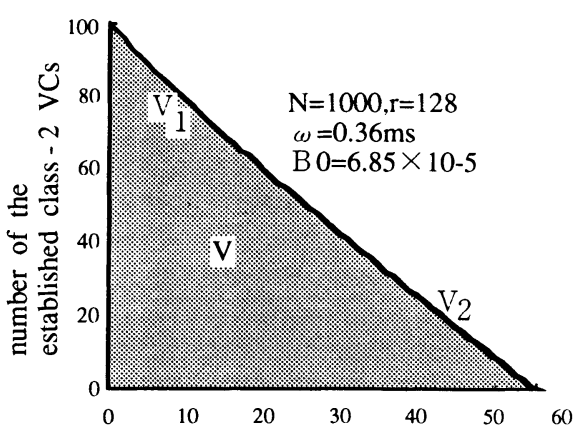

number of the established class - 1 VCs

VP bandwidth: $150 \mathrm{Mb} / \mathrm{s}$

class peak cell rate average cell rate

$1 \quad 9 \mathrm{Mb} / \mathrm{s} \quad 2.4 \mathrm{Mb} / \mathrm{s}$

$21 \mathrm{Mb} / \mathrm{s} \quad 1.2 \mathrm{Mb} / \mathrm{s}$

Fig. 8. Region that can admit VCs .

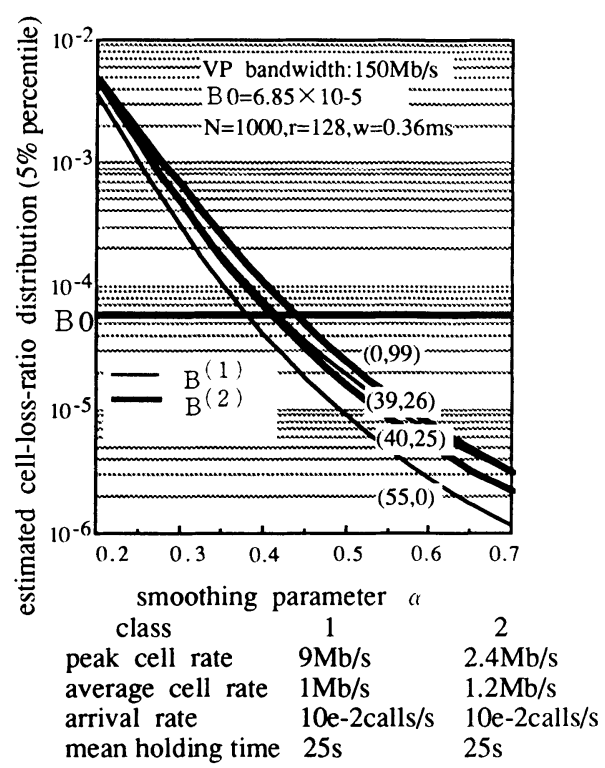

Fig. 9. The 5\% percentile point of the estimated cell-loss-ratio distribution. 


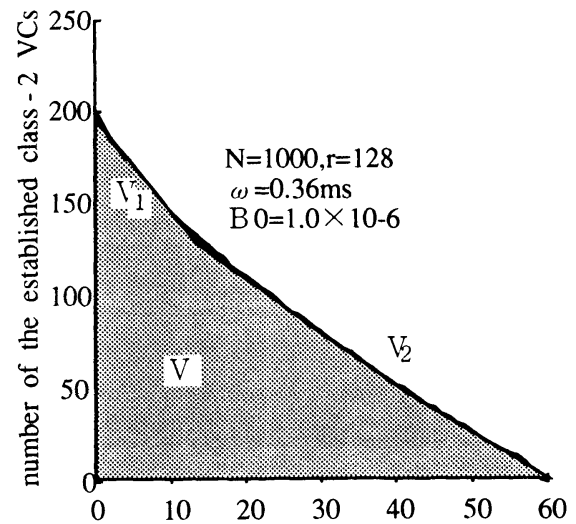

number of the established class - 1 VCs

VP bandwidth: $150 \mathrm{Mb} / \mathrm{s}$

class peak cell rate average cell rate

$\begin{array}{ccc}1 & 12 \mathrm{Mb} / \mathrm{s} & 1.2 \mathrm{Mb} / \mathrm{s} \\ 2 & 0.6 \mathrm{Mb} / \mathrm{s} & 0.6 \mathrm{Mb} / \mathrm{s}\end{array}$

Fig. 10. Region that can admit VCs .

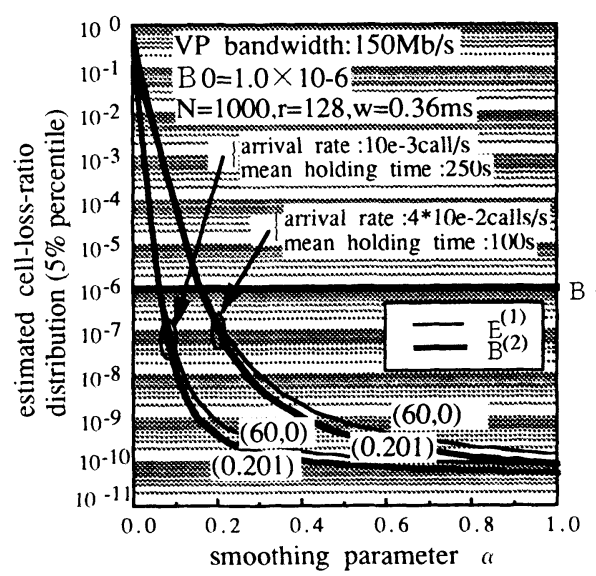

class

peak cell rate $\quad 12 \mathrm{Mb} / \mathrm{s} \quad 1.2 \mathrm{Mb} / \mathrm{s}$

average cell rate $0.6 \mathrm{Mb} / \mathrm{s} \quad 0.6 \mathrm{Mb} / \mathrm{s}$

Fig. 11. The $5 \%$ percentile point of the estimated cell-loss-ratio distribution.

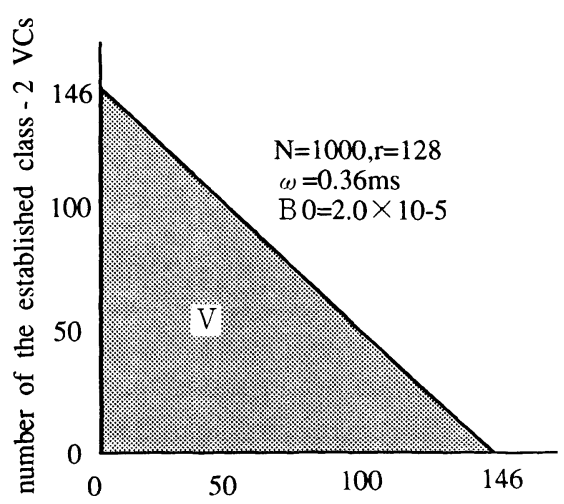

number of the established class - 1 VCs

VP bandwidth:200Mb/s

class peak cell rate average cell rate

$\begin{array}{ccc}1 & 5 \mathrm{Mb} / \mathrm{s} & 5 \mathrm{Mb} / \mathrm{s} \\ 2 & 1 \mathrm{Mb} / \mathrm{s} & 1 \mathrm{Mb} / \mathrm{s}\end{array}$

Fig .12. Region that can admit VCs . (VP bandwidth $200 \mathrm{Mb} / \mathrm{s}$ )

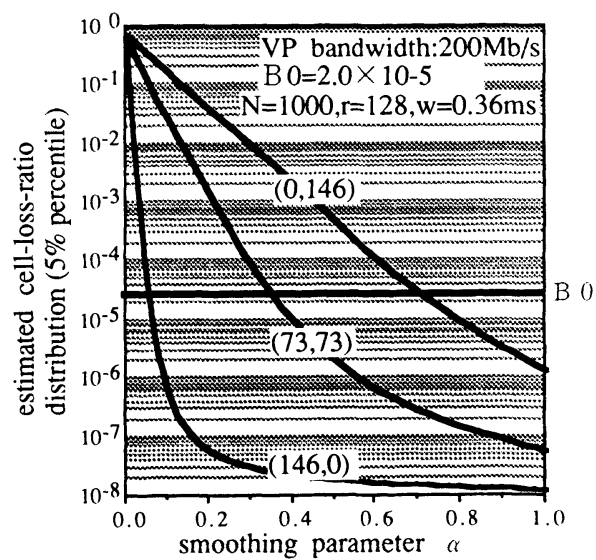

class

peak cell rate $\quad 5 \mathrm{Mb} / \mathrm{s} \quad 5 \mathrm{Mb} / \mathrm{s}$

average cell rate $1 \mathrm{Mb} / \mathrm{s} \quad 1 \mathrm{Mb} / \mathrm{s}$

arrival rate $10 \mathrm{e}-3 \mathrm{calls} / \mathrm{s} \quad 10 \mathrm{e}-2 \mathrm{calls} / \mathrm{s}$

mean holding time $250 \mathrm{~s} \quad 25 \mathrm{~s}$

Fig. 13. The $5 \%$ percentile point of the estimated cell-loss-ratio distribution .

(VP bandwidth $200 \mathrm{Mb} / \mathrm{s}$ ) 

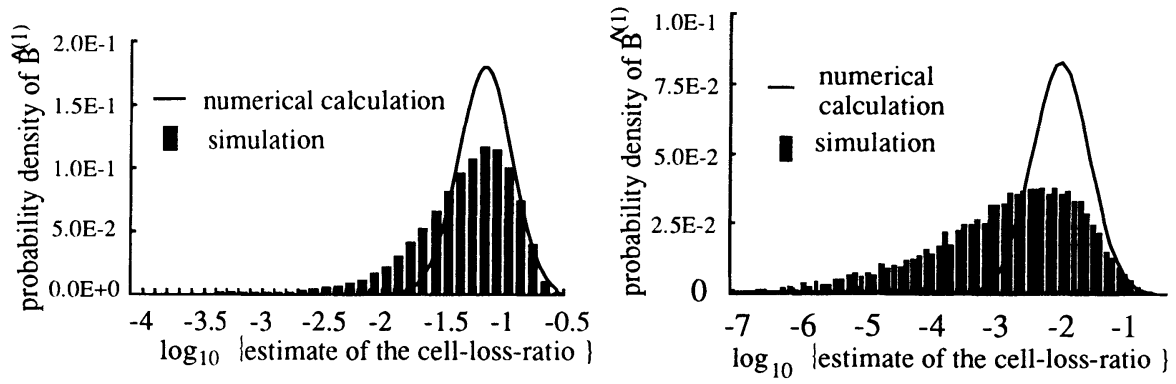

$<$ traffic condition $>$

$<$ traffic condition $>$

$\mathrm{C}=150 \mathrm{Mb} / \mathrm{s}, \mathrm{N}=100, \mathrm{r}=64, \mathrm{w}=0.18 \mathrm{~ms}$, $\mathrm{m}=1, \alpha=0.14$,

$\mathrm{R} 1=7 \mathrm{Mb} / \mathrm{s}, \mathrm{Al}=3.5 \mathrm{Mb} / \mathrm{s}, 1 / \lambda \mathrm{l}=5 \mathrm{~s}$,

$\mathrm{L} 1=0.05 \mathrm{~s}, \mathrm{nl}=40$

Fig. 14 Probability density of $\hat{B}^{(1)}(40)$. $\mathrm{C}=150 \mathrm{Mb} / \mathrm{s}, \mathrm{N}=100, \mathrm{r}=64, \mathrm{w}=0.18 \mathrm{~ms}$, $\mathrm{m}=2, \alpha=0.5$,

$\mathrm{R} 1=14 \mathrm{Mb} / \mathrm{s}, \mathrm{A} 1=3.5 \mathrm{Mb} / \mathrm{s}, 1 / \lambda 1=0.5 \mathrm{~s}$, $\mathrm{L} 1=0.05 \mathrm{~s}, \mathrm{n} 1=13$,

$\mathrm{R} 2=7 \mathrm{Mb} / \mathrm{s}, \quad \mathrm{A} 2=3.5 \mathrm{Mb} / \mathrm{s}, \quad 1 />2=5 \mathrm{~s}$

$\mathrm{L} 2=0.5 \mathrm{~s}, \mathrm{n} 2=12$

Fig. 15. Probability density of $\widehat{B}^{(1}(13,12)$. 